objective of this study was to evaluate the risk factors that predispose IT professionalsto the development of WRMSD.

Methods A prospective analysis of 7280 employees of a single IT company in an Industrially Developing Country was conducted. Among them, 5210 were males and 2070 were females, between the ages 20 to 60 years. The employees were evaluated by a detailed questionnaire consisting of demographic data, job details, health status, physical risk factors, short-form Work Style Questionnaire and Nordic Musculoskeletal Pain Questionnaire. The data were extracted and statistical analysis was done.

Result The mean age of the employees was 32.5 years.58\% of the employees were diagnosed by an experienced occupational health physician (OHP) to have a WRMSD, which predominantlyincluded myofascial pain syndrome of the neck, upper and lowerback. Age, Body Mass Index (BMI), working hours and work-style were positively correlated $(\mathrm{r}<0.01)$ with the presence of WRMSDs, as higher the age and BMI, increased working hours and higher work-style score showed higher prevalence of WRMSD. On the other hand,rest breaks during work, regular exercises and formal ergonomics training were negatively correlated $(\mathrm{r}<-0.01)$ with the presence of WRMSDs, as more frequent breaks, regular exercises and ergonomics training showed lower prevalence of WRMSD. Also, the presence of co morbidities like diabetes, hypothyroidism and osteopenia/osteoporosis had a positive influence on the prevalence of WRMSDs in the study population. Other specific factors like work experience, hand dominance, type of computer used also had an influence on the development of WRMSDs.

Discussion The risk factor analysis gives an insight to the appropriate areas of ergonomic interventions among IT professionals.

\section{ROTATING SHIFT WORK AND METABOLIC SYNDROME COMPONENTS AMONG WORKERS AT AN ELECTRICITY DISTRIBUTION COMPANY IN ISMAILIA CITY, EGYPT}

${ }^{1}$ Hebatalla M Aly*, ${ }^{1}$ Ayman E Fahim, ${ }^{2}$ Mohamed M Elshabrawy, ${ }^{1}$ Ashraf M Elshatawy, ${ }^{1}$ Amani W Abd El-Halim Abdallah. ${ }^{1}$ Department of Community Medicine; Faculty of Medicine; Suez Canal University; Ismailia, Egypt; ${ }^{2}$ Department of Clinical Pathology; Faculty of Medicine; Suez Canal University; Ismailia, Egypt

\subsection{6/oemed-2018-ICOHabstracts.423}

Introduction and aim Shift work is increasing nowadays. More than fifth employees are shift workers in industrialised countries. The body's biological cycles circadian rhythms that recur at 24 hour intervals is disturbed. This includes sleep-wake patterns, body temperatures and hormone levels. Feeding behaviour, lipid and carbohydrate metabolism and blood pressure are also subjected to daily variation. Studies have shown that rotating shift work has high propensity to metabolic and nutritional disorders and may be directly responsible for increased obesity, higher blood pressure levels, altered nutritional metabolism, insulin resistance, diabetes, dyslipidemias, gastrointestinal disorders and metabolic syndrome. This work was aimed at clarifying the relation between rotating shift work and metabolic syndrome components for better health and well-being of rotating shift workers.

Methods A comparative study (ex- post facto design) was carried out in an Electricity Distribution Company in Ismailia city, Egypt. One hundred eligible shift workers and 100 non shift workers were chosen by systematic random method. The updated IDF criteria for diagnosing metabolic syndrome was used (International Diabetes Federation IDF, 2006). All participants were subjected to interview questionnaire, weight, height, waist circumference and blood pressure measurements, fasting blood sugar level, serum triglycerides and HDL levels.

Results According to IDF criteria, 53\% of shift workers had metabolic syndrome compared to $39 \%$ of day workers $(\mathrm{p}=0.04)$ with odds ratio 1.8 . The mean number of components affecting shift workers was higher than day workers $(2.7$ \pm 1.3 versus $2.2 \pm 1.4$ respectively; $p=0.00$ ). Regarding each component; central obesity, blood pressure and blood sugar were significantly higher among shift workers compared to day workers. Triglycerides and HDL didn't differ significantly between the 2 groups. Shift workers were also suffering more from sleep disorders (insomnia, snoring or obstructive sleep apnea) compared to day workers (55\% versus 39\% respectively; $\mathrm{p}=0.02)$. Using a dietary assessment score, unhealthy dietary habits were significantly more compared to day workers $(6.7 \pm 7.2$ versus $4.4 \pm 5.9$ respectively; $p=0.01)$.

Conclusion Metabolic syndrome are higher among shift workers compared to day workers. Also some components of metabolic syndrome as central obesity, blood pressure and blood sugar affect shift workers more than day workers.

\section{OCCUPATIONAL MUSCULOESKELETAL DISORDERS AS A RISK FACTOR FOR USING HYPNOTICS: AN STUDY BASED ON 2013 BRAZILIAN NATIONAL HEALTH SURVEY (PNS)}

Fernando Feijo, Gustavo Jaeger, Luís Paulo Ruas, Maria Del Pilar Quispe, Nadege Jacques, Fernando Wehrmeister. Federal University Of Pelotas, Pelotas, Rio Grande do Sul, Brazil

\subsection{6/oemed-2018-ICOHabstracts.424}

Introduction Hypnotics are one of the most prescribed drugs in the world and are related to several morbidity and mortality outcomes. Some risk factors such as sex, age, marital status, and also chronic pain e mental disorders have been described in medical papers. However, there are restrict data concerning the influence of occupational factors in the use of sleep drugs. Therefore this study aimed to evaluate the association between MSD and use of hypnotics in the Brazilian population.

Methods Cross-sectional study with secondary data from the PNS 2013. We used descriptive statistics to present exposure and outcome variables. Chi-square was proceeded to test differences between groups and Logistic Regression controlling for covariates was made to analyse the main association.

Results The general prevalence of hypnotics use was 7,1\%. The prevalence was higher in women and in elder people, increasing according to the age. The general prevalence of MSD was 1,9\%. Individuals with MSD presented 2 times higher prevalence of hypnotics use compared to controls, even after adjusting for covariates.

Disscussion When we consider the problem of the use of medication to sleep, it should be attempted to MSD as a possible risk factor and focus of intervention. New researches are necessary to better elucidate the role of musculoskeletal disorders in the hypnotics use. 